\title{
Simulative Investigation of Long- Range 32x3 Gbps WDM-FSO Link Under Different Weather Attenuation Conditions
}

\author{
Gurpreet Kaur $^{1}$, Harmeet Singh ${ }^{2}$, Amandeep Singh Sappal ${ }^{3}$ and Manpreet Kaur ${ }^{4}$ \\ ${ }^{1}$ Mtech scholar (ECE), CTIEMT (PTU), India-1441001 \\ ${ }^{2}$ Assistant Professor, Department of ECE, CTIEMT (PTU), India-1441001 \\ ${ }^{3}$ Assistant Professor, Department of ECE, Punjabi University, India-147002 \\ 1parmargurpreet18@gmail.com, ${ }^{2}$ harmeetsingh85.hs@gmail.com \\ ${ }^{3}$ Sappal73as@yahoo.co.in
}

\begin{abstract}
Objective: To design and investigate long range efficient 32-channel WDM-FSO system under worst weather conditions at data rate of $3 \mathrm{~Gb} / \mathrm{s}$. Methods/Statistical Analysis: In this paper, 32 channel WDM-FSO system has been designed and investigated under worst weather conditions using NRZ modulation at $3 \mathrm{~Gb} / \mathrm{s}$. Opti-system software is used to simulate the system. Findings: In this paper, the WDM-FSO system investigated under worst weather conditions using NRZ modulation in terms of Bit error rate and quality factor at $3 \mathrm{~Gb} / \mathrm{s}$ and also achieved the maximum possible link range with minimum target value of Bit Error Rate (BER) by using semiconductor optical amplifier. The present system supports the transmission up to $431 \mathrm{~km}$ that is achieved under clear weather condition at BER of $10^{-10}$. Application/Improvements: Results showed the maximum link range under worst channel conditions with minimum acceptable bit error rate By using the SOA pre-amplification at receiver side.
\end{abstract}

Keywords: Free Space Optics, Bit Error Rate, semiconductor optical amplifier, and wavelength division multiplexing

\section{Introduction}

Optical networks are high capacity networks that use a light as an electromagnetic carrier wave modulated to transmit data these systems offer excellent automatic powerlevel control, and eliminate short-distance optical saturation [1]. Free Space Optics (FSO) is a communication technology that uses light as carrier and free space as medium to transfer information between transmitter and receiver separated by certain distance. FSO link is a wireless link between a transmitter and a receiver in optical communication system. The data which has to be transmitted is modulated on the intensity, phase and frequency of the carrier signal [2]. In FSO system different modulation techniques are used to modulate information signal at source. Each FSO system uses a high-power optical transmitter for transmit source signal towards destination and receiving side high sensitivity receiver used. But the atmospheric attenuation is major challenge for faced by FSO systems which affect the performance of the link. The other factors which can affect the FSO are humidity, water-vapors, signals absorption, smoke, beam scintillation, spreading and wandering are some of the factors [3]. FSO systems operate in the infrared (IR) range of spectrum. It desires an unobstructed line-of-sight between the transmitter and receiver for proper operation of an FSO system. FSO systems operates around 850 and $1550 \mathrm{~nm}$ wavelengths and the frequencies corresponding to this range of wavelengths is around $200 \mathrm{THz} .1550 \mathrm{~nm}$ wavelength is preferred because of more eye safety and reduced solar background radiation. This system do not require any license fees. So, it is easily affordable. Moreover, they can be installed much more quickly. [4]. 
The main aim of every communication system is to increase the transmission distance and speed. In the field of FSO communication, the wavelength division multiplexing (WDM) is one of the efficient techniques used to provide high-capacity long distance transmission [5]. The block diagram of WDM-FSO system is shown in Figure 1.

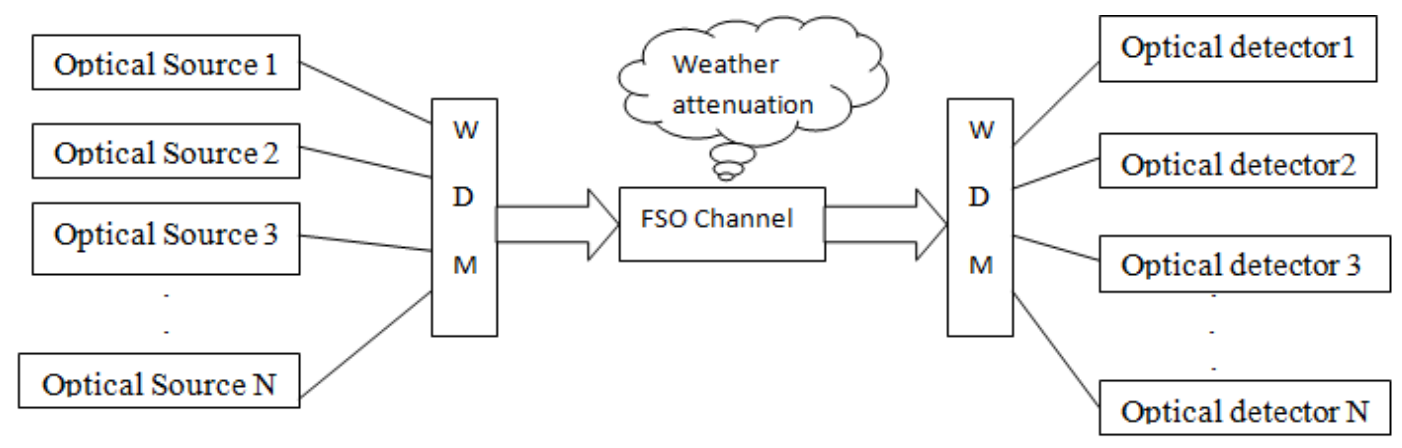

Figure 1. FSO-WDM System

It is a technique in which multiple signals are transmitted over single channel. WDM technique becomes most popular due to the fact that capacity of system can be increased by increasing the number of channels and tapering the channel spacing without using more than one FSO link. So WDM approach can be applied in FSO systems to maximize bandwidth usage in cheaper way [6]. WDM system is designed to conquer the problem of FSO signal degradation due to atmospheric disorder. WDM system has high capacity so that it has ability to achieve high data rate and longer link distance due to more laser power [3]. The amplification of optical signal can be done by optical amplifiers such as RAMAN, EDFA or SOA. In this paper SOA is used for amplification of optical signal because it has unique properties such as Size of SOA is compact as compared to erbium doped fiber amplifiers (EDFAs) and Raman optical Amplifiers, High-speed capability, low switching energy. SOA amplifiers have Large BW \& can operate at 800,1300 , and $1500 \mathrm{~nm}$ wavelength regions. All these properties craft SOA a good choice for optical amplification [7] [8]. Laser power has plays important role in designing any system; so lesser this value, higher will be the quality of system [1].

In this paper, the work has been extended from work reported in [1], [2] by presenting less input power requirement, an improved bit error rate (BER) and Q factor by using efficient optical amplifier under worst weather condition. From the results, it has been analyzed that by using SOA as amplifier, the system performs better in terms of bit error rate and quality factor with increased link distance as compared to [1] and [2].

The rest of the paper is organized as follows: Section 2 described the simulation setup. In Section 3 simulation results and discussions have been reported and conclusions are given in Section 4.

\section{Simulation Setup}

The simulation design of 32-channel WDM-FSO communication system with 100 $\mathrm{GHz}$ channel spacing is as shown in Figure 2. The transmitter section consists of PseudoRandom Bit Generator, NRZ Pulse Generator, CW Laser array, WDM multiplexer and Mach-Zehnder Modulator. Binary sequence of data converted to electrical signal by NRZ Pulse generator. The outputs of different optical source are collected by WDM Multiplexer. The signals from data source and WDM-MUX are applied to the MachZehnder modulator which modulates the intensity of optical signals from the optical source array. The output signal of modulator is transmitted over FSO link under different weather attenuation conditions. On the receiver side firstly the SOA pre-amplification is done to raise the signal strength then WDM-Demultiplexer is used to separate the 
different signals. Select selection is used next to the output of WDM demux. APD photo detector is used to detect the optical signal and converted into electrical form. Now Low pass Bessel filter is used to filter the signal and then to visualize the signal a BER analyzer is used. With proper parameters, WDM based FSO system can be optimized to achieve a maximum link range of operation. These system design parameters are given in Table 1. The quality of the received signal is considerably depends on the conditions of the FSO channel and the WDM system design.

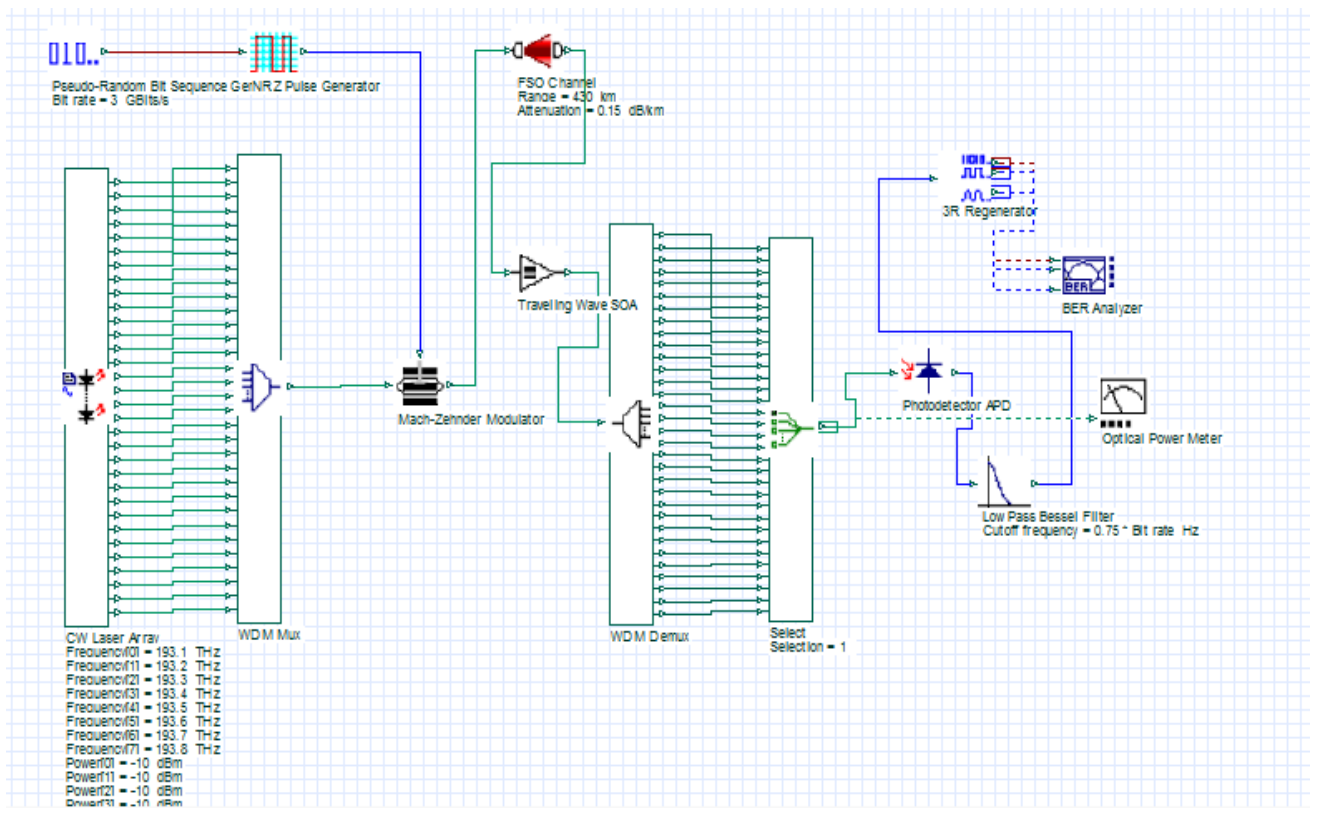

Figure 2. Simulation Setup of 32-channel WDM-FSO Communication System

Table 1. Simulation Parameters

\begin{tabular}{|c|c|}
\hline Parameter & Value \\
\hline Bit rate & $3 \mathrm{Gbps}$ \\
\hline Laser wavelength $(\lambda)$ & $1550 \mathrm{~nm}$ \\
\hline CW array laser frequency & $193.1-196.2 \mathrm{THz}$ \\
\hline Transmitter aperture diameter & $10 \mathrm{~cm}$ \\
\hline Receiver aperture & $20 \mathrm{~cm}$ \\
\hline Beam divergence & $1 \mathrm{mrad}$ \\
\hline
\end{tabular}

\section{Simulation Results and Discussions}

This paper presents simulated results to evaluate the performance of 32 channel WDM-FSO communication link at $3 \mathrm{Gbps}$ data rate under worst weather conditions. The performance analysis of system under very clear, clear, light haze, heavy haze, light rain and heavy rain weather conditions are shown in Table 2. The results show that Maximum link distance is achieved at acceptable minimum BER values for different atmospheric conditions with optimized values of laser power and data rate. It has been observed that as increase in attenuation causes reduce in the transmission link distance with acceptable BER and Q-factor. It can be seen that for clear weather condition the maximum link can be carried out up to $431 \mathrm{~km}$ while it get reduced to $40.2 \mathrm{~km}$ for heavy haze condition and $6.147 \mathrm{~km}$ for heavy rain condition with BER of 4.03891e-10, 6.36369e-10and 8.16576e- 
10 respectively. The eye opening and BER for the clear, light haze, heavy haze, light rain and heavy rain are seen in Figure 3. Large eye opening means less BER and good communication.

Table 2. Performance Analysis of System under Different Weather Conditions

\begin{tabular}{|c|c|c|c|c|c|}
\hline $\begin{array}{c}\text { Weather } \\
\text { Condition }\end{array}$ & $\begin{array}{c}\text { Attenuation } \\
(\mathbf{d B} / \mathbf{k m})\end{array}$ & $\begin{array}{c}\text { Laser Power } \\
(\mathbf{d B m})\end{array}$ & $\begin{array}{c}\text { Max. Link } \\
(\mathbf{K m})\end{array}$ & BER & Q-Factor \\
\hline Very clear & 0.15 & -10 & 431 & $4.03891 \mathrm{e}-10$ & 6.14315 \\
\hline Clear & 0.299 & -10 & 234 & $4.52266 \mathrm{e}-10$ & 6.12540 \\
\hline Light haze & 0.61 & 10 & 153.8 & $5.75255 \mathrm{e}-10$ & 6.08627 \\
\hline Heavy haze & 2.62 & 10 & 40.2 & $6.36369 \mathrm{e}-10$ & 6.07005 \\
\hline Light rain & 6.80 & 10 & 16.66 & $7.61924 \mathrm{e}-10$ & 6.04143 \\
\hline Heavy rain & 19.77 & 10 & 6.147 & $8.16576 \mathrm{e}-10$ & 6.03033 \\
\hline
\end{tabular}

Eye diagrams for different weather conditions are shown below:

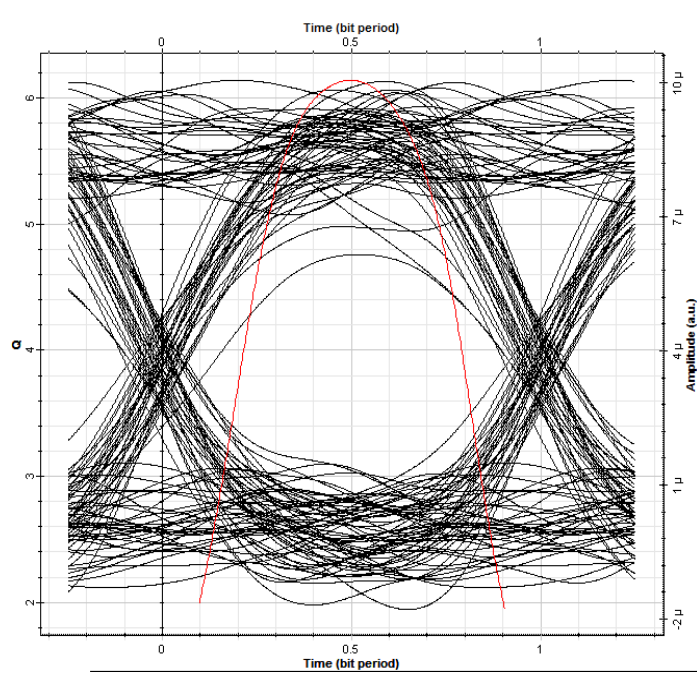

(a)

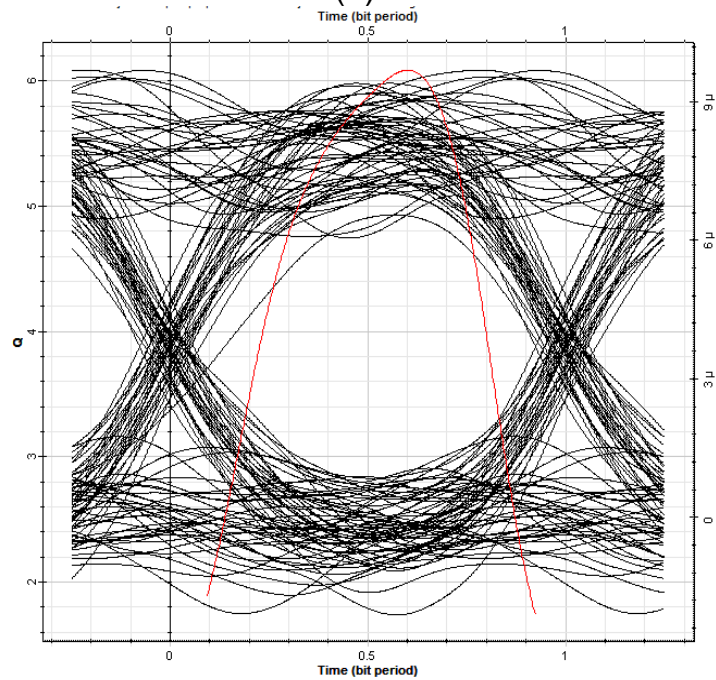

(c)

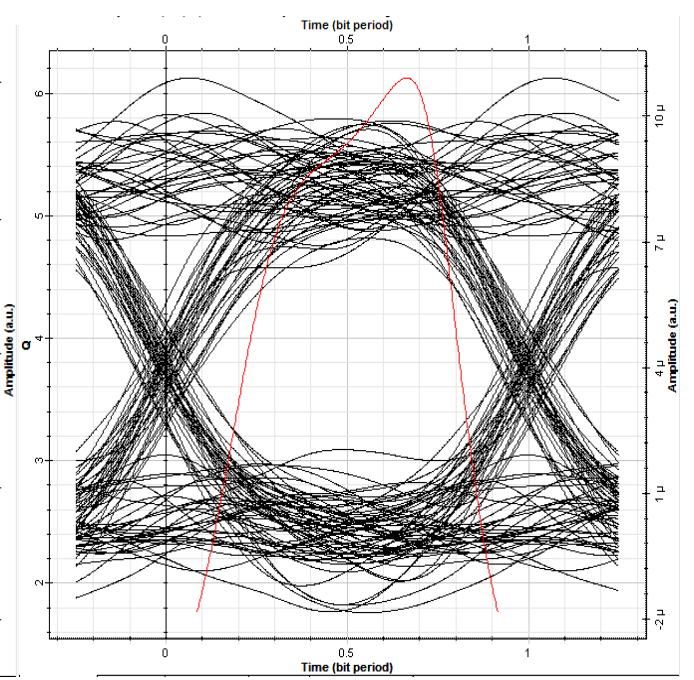

(b)

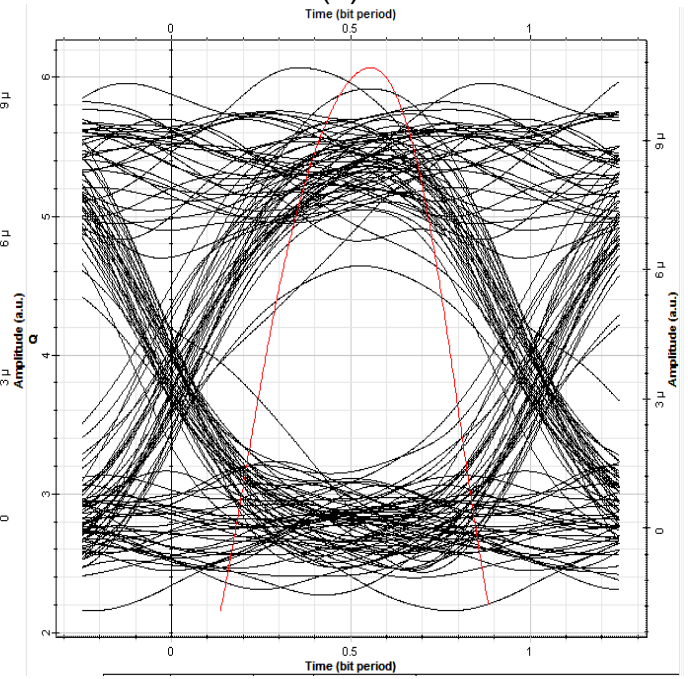

(d) 


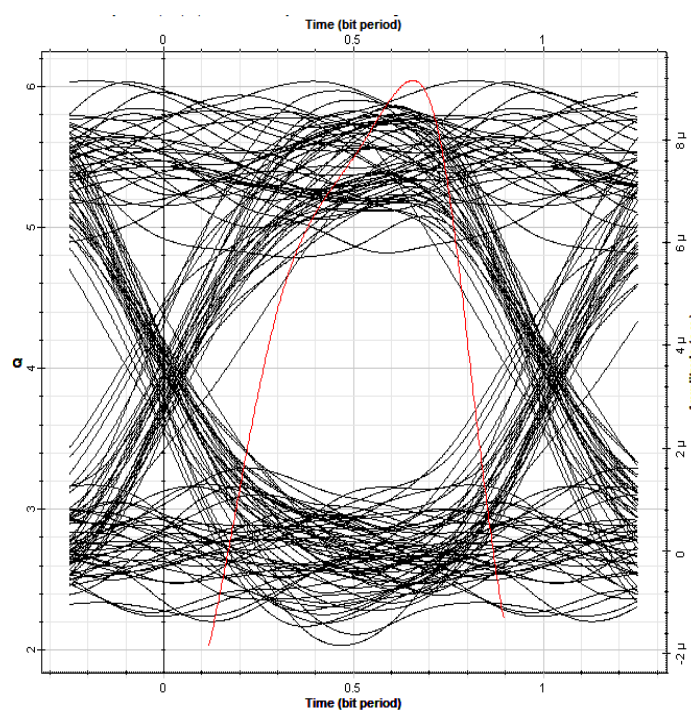

(e)

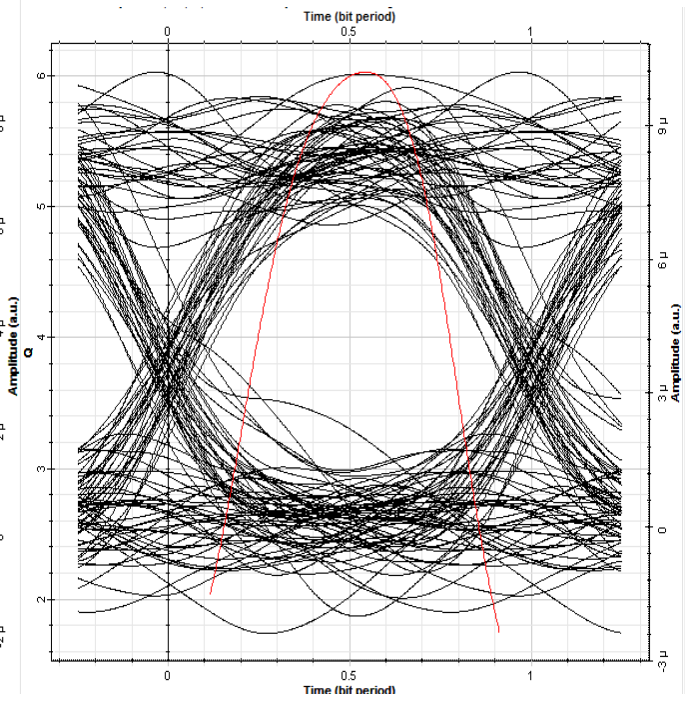

(f)

Figure 3. Eye Diagrams for Different Weather Conditions: (a) Very clear sky (b) Clear Sky (c) Light Haze (d) Heavy Haze (e) Light Rain \& (f) Heavy Rain

Figure 4 shows that Max. Q-factor v/s link distance under different weather conditions. The increase in the attenuation leads to decreasing in the link range. The acceptable Qfactor is 6.03033 and 6.14315 for heavy rain and very clear weather at the maximum link range. It can also be observed that with increase in attenuation (worse weather condition), $\mathrm{Q}$ factor decreases.

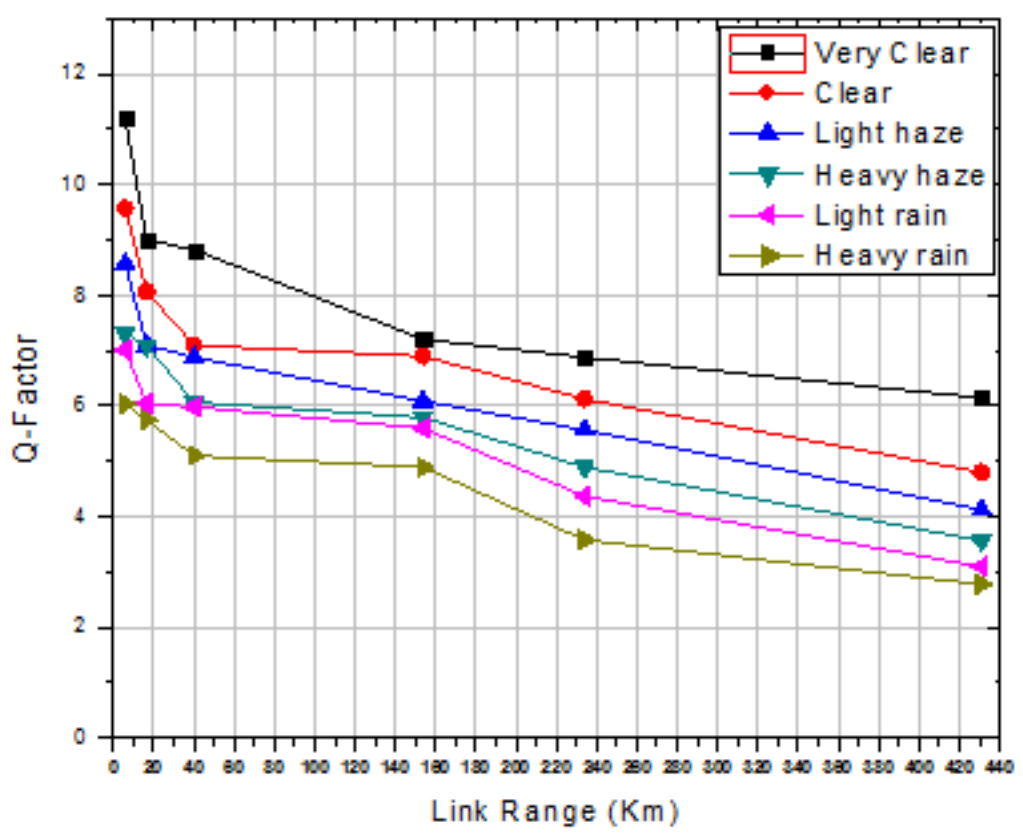

Figure 4. Q-factor v/s Range

Figure 5 shows the graph of the BER v/s link range under different weather conditions. From the graph, it has been observed that the sustainable distance up to $431 \mathrm{~km}, 234 \mathrm{~km}$, $153.8 \mathrm{~km}$ and $40.2 \mathrm{~km}$ is achieved with BER of $10^{-10}$ for very clear, clear, light haze and 
heavy haze respectively. The graph shows that the BER increases with increase in attenuation as offered by weather conditions. For strong attenuation (worst weather condition) the link is limited to $16.66 \mathrm{~km}$ and $6.147 \mathrm{~km}$ for light rain and heavy rain respectively with BER of $10^{-10}$.

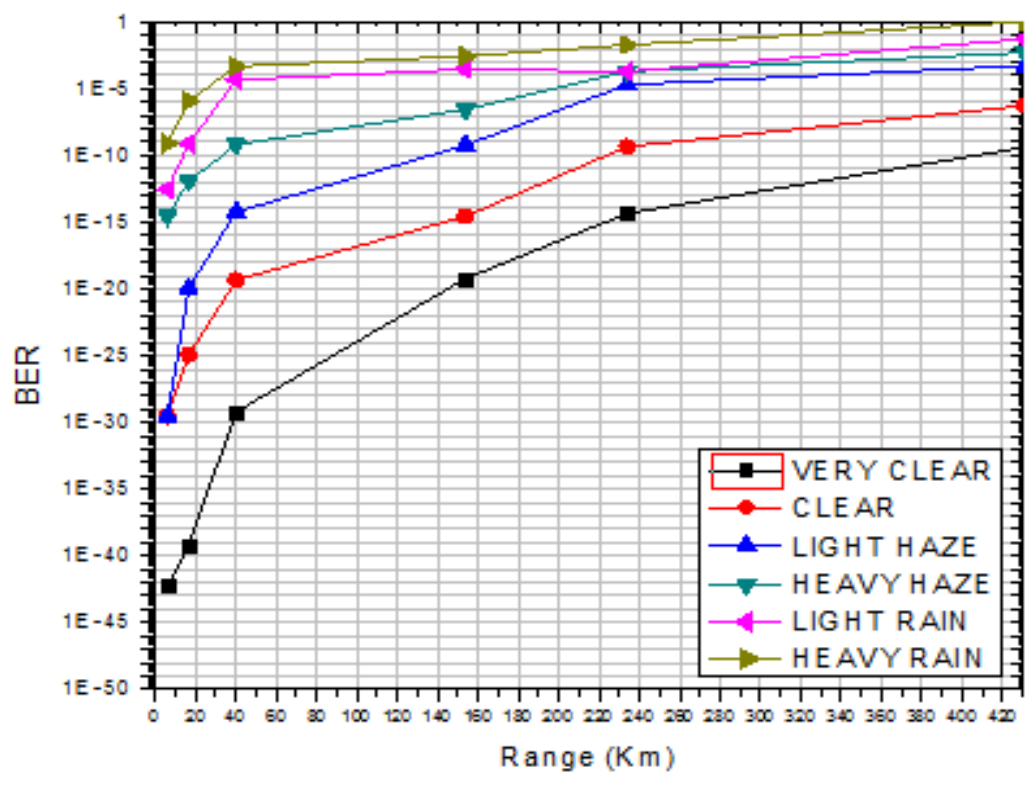

Figure 5. BER v/s Link Range

\section{Conclusion}

This paper emphasizes on the simulation design and investigation of 32 channels WDM-FSO communication link at $3 \mathrm{~Gb} / \mathrm{s}$ using digital data format (NRZ modulation technique) and performance is explored under improved optimized parameters. The performance of simulated system has been investigated in terms of BER and Q-factor as shown in graphs above. From simulation results, it has been observed that the WDM-FSO system can work up to $431 \mathrm{~km}$ with BER of $10^{-10}$ by using SOA amplification. With increase in atmospheric attenuation as the weather condition gets inferior; the maximum achieved distance can be extended up to $6.147 \mathrm{~km}$ and $40.2 \mathrm{~km}$ for heavy rain condition and heavy haze condition with minimum BER of 8.16576e-10and 6.36369e-10.

\section{References}

[1] Aditi and Preeti, "An effort to design a power efficient long reach WDM-FSO system", Proceeding of IEEE, International Conference on Signal Propagation and Computer Technology, (2014).

[2] Mazin Ali A. Ali, "Performance analysis of terrestrial WDM-FSO Link under Different Weather Channel", Journal (World Scientific News), Vol. 56, November (2016).

[3] H. Hilal A. Fadhil, A. Amphawan and N. Ahmed, "Optimization of free space optics parameters: An optimum solution for bad weather conditions “, Elsevier, Optik 124(2013), pp 3969-3973.

[4] [Online].Available:http://electronicsforu.com/technology-trends/fourth-generation-free-space-optics.

[5] H. Singh and M. Arora, "Investigating Wavelength Dependency of Terrestrial Free Space optical Communication Link", International journal of Scientific Research in Science and Technology, Vol. 2, May (2016), no. 2395-6011.

[6] S.parkash, A.Sharma, H.Singh and H.P Singh, "performance investigation of 40Gb/s DWDM over free space optical communication system using RZ modulation format", advances in optical technologies, Vol.2, (2016), article id 4217302, pp. 8.

[7] R.Jee and S.Chandra, "Performance Analysis of WDM-Free-Space Optical Transmission system with MQAM Modulation under Atmospheric and optical nonlinearities" in Proceeding of IEEE, International Conference on microwave, optical and Communication engineering, IIT Bhubaneswar, India,(2015). 
[8] C. Sharma, S. Singh, B. Sharma, "Investigations on Bit Error Rate Performance of DWDM Free Space Optics System Using Semiconductor Optical Amplifier in Intersatellite Communication”, International Journal of Engineering Research \& Technology, Vol. 2 ,Issue 8, August ( 2013).

[9] Mazin Ali A. Ali, "Comparison of NRZ, RZ-OOK Modulation Formats for FSO Communications under Fog Weather Condition”, International Journal of Computer Applications, Vol.108, no. 2, ( 2014).

[10] M. A. Esmail, H. Fathallah and M. S. Alouni, "Outdoor FSO Communications Under Fog: Attenuation Modeling and Performance Evaluation”, IEEE Photonics Journal, Vol. 8, no. 4, (2016).

[11] M. A. Esmail, H. Fathallah and M. S. Alouni, "analysis of fog effects on terrestrial free space optical communication links", IEEE International Conference on Communications Workshops (ICC), Kuala Lumpur, Malaysia, (2016). 
International Journal of Advanced Science and Technology Vol.102 (2017) 